\title{
Finickiness in VMH rats also results from the lesions, not just from obesity
}

\author{
RONALD H. PETERS and MARK W. GUNION \\ Iowa State University, Ames, Iowa 50011
}

\begin{abstract}
When a high-fat diet was successively adulterated with increasing concentrations of quinine, from $.4 \%$ to $4.0 \%$, the mean body weight of female rats with ventromedial hypothalamic (VMH) lesions never fell below that of same-diet control rats. In a second experiment, when female VMH-lesioned and control rats were maintained on a 1.6\% quinine high-fat diet for the first 15 days after surgery, VMH rats ate less and lost more weight than same-diet control rats before they displayed typical large weight gains when they were subsequently fed the unadulterated high-fat diet. Diet palatability interacts with VMH destruction to alter both the upper and lower limits of body-weight maintenance.
\end{abstract}

Rats with lesions of the ventromedial hypothalamus (VMH) are generally described as finicky eaters because they overrespond to the palatability of their diets (Miller, Bailey, \& Stevenson, 1950; Teitelbaum, 1955). Enhanced food intake and body-weight gain occur when VMH rats are fed standard laboratory diets that are considered reasonably palatable. When diet palatability is decreased by adulteration with substances such as quinine, VMH rats reject these diets more readily than do intact animals.

Following the initial demonstrations of the effects of palatability manipulations on the feeding behavior of VMH rats, several researchers argued that obesity, rather than VMH destruction per se, induces finickiness because VMH rats maintained their body weights at control levels when fed quinine-adulterated maintenance diets (Ferguson \& Keesey, 1975; Franklin \& Herberg, 1974). Finickiness was defined as undereating and weight loss relative to values for same-diet control rats rather than simply the failure to overeat and gain weight.

We recently reported data that both challenge and yet support the concept of VMH finickiness (Peters, Luttmers, Gunion, \& Wellman, 1978). Rats with VMH lesions maintained for 30 days on a high-fat diet adulterated with $.4 \%$ quinine not only ate and weighed more than same-diet control rats but outgained control rats fed an unadulterated high-fat diet as well. Subsequent reports from this laboratory demonstrated that $\mathrm{VMH}$ rats ate and gained more than same-diet control rats when their ground-chow maintenance diet was adulterated with either $50 \%$

This research was supported in part by an Iowa State University Graduate College research grant to M.W.G. The assistance of Anne Kennedy during Experiment 2 is gratefully acknowledged. A preliminary report of this work was presented at the annual meeting of The Psychonomic Society, San Antonio, 1978. Requests for reprints should be sent to R.H.P. cellulose (Wellman \& Peters, 1978) or .2\% quinine (Peters, Wellman, \& Gunion, 1979). In each of the above instances, control rats fed the adulterated diet ate and weighed less than control rats fed the unadulterated diet. Thus, in all three instances, VMH rats overate a diet whose palatability was substantially decreased as indexed by the feeding behavior of intact rats.

In support of the commonly held notion that VMH lesions induce finickiness, we showed that rats with VMH lesions that would subsequently become obese when fed an unadulterated diet initially underate and lost weight relative to same-diet control rats when maintained on a $.2 \%$ quinine wet-mash diet. Surprisingly, this was and is currently the only demonstration of finickiness in rats with VMH lesions defined as undereating and weight loss relative to values for control rats fed the same diet. Undereating, under these circumstances, is obviously not confounded with obesity. There are three reports (Gale \& Sclafani, 1977; Peters, Wellman, \& Gunion, 1979; Sclafani, Springer, \& Kluge, 1976) which indicate that rats with knife cuts between the medial and lateral hypothalamus weigh less than same-diet control rats when fed quinine-adulterated diets, but Peters et al. (1979) report that rats with hypothalamic knife cuts do not display patterns of feeding behavior identical to those of VMH-lesioned rats during various palatability manipulations.

The purpose of the present study was to obtain further evidence on the issue of undereating and weight loss in rats with VMH lesions relative to same-diet control rats. Since we had obtained the only positive evidence using a $.2 \%$ quinine wet-mash diet and had also demonstrated that VMH-lesioned rats would overeat a $.4 \%$ quinine high-fat diet, we chose to adulterate the high-fat diet with even higher concentrations of quinine to further decrease its palatability. Experiment 1 was an initial search for effective con- 
centrations, and Experiment 2 demonstrated that rats with VMH lesions ate and weighed significantly less than same-diet control rats when their high-fat diet was adulterated with $1.6 \%$ quinine.

\section{EXPERIMENT 1}

\section{Method}

Subjects. The subjects were 12 female Long-Evans hooded rats (Blue Spruce Farms, Inc.) weighing 247 to $309 \mathrm{~g}$ at the beginning of the experiment. They were housed singly in suspended wiremesh cages in an isolated room $\left(24^{\circ} \mathrm{C}\right)$ under a $12 / 12$-h light/dark cycle. They had continuous access to tap water and the diets described below.

Diets. The basic high-fat diet consisted of $2 / 3$ powdered laboratory chow (Purina) and 1/3 melted vegetable shortening (Crisco). This diet was adulterated successively with $.4 \%, .6 \%, .8 \%, 1.2 \%$, $1.6 \%, 2.4 \%$, and $4.0 \%$ quinine sulfate $(\mathrm{w} / \mathrm{w})$. Fresh food was given every 3 days in 87 -mm-high $\times 70$-mm-diam wide-mouth jars held in position by a wire loop attached to the front of the cage. The animals were weighed at the end of each of these 3-day periods.

Surgery. Rats received either bilateral VMH lesions $(n=6)$ or sham surgery $(n=6)$. Surgery was performed under secobarbital anesthesia (Myothesia, $42 \mathrm{mg} / \mathrm{kg}$, i.p.) with methyl atropine $(10 \mathrm{mg} / \mathrm{kg}$, i.p.) given to decrease respiratory complications. VMH lesions (Group V) were made using a 30-ga nichromestainless steel electrode insulated except for $.5 \mathrm{~mm}$ at the conical tip. The tip of the electrode was positioned $.5 \mathrm{~mm}$ above the base of the brain, $5.8 \mathrm{~mm}$ anterior to the interaural line, and $.7 \mathrm{~mm}$ lateral to the midline, with the upper incisor bar $5.0 \mathrm{~mm}$ above the interaural line. Lesions were produced by passing $2.0 \mathrm{~mA}$ anodal current for $20 \mathrm{sec}$ between the electrode and rectal cathode. Sham surgery (Group C) was performed by anesthetizing the rat, incising the scalp, and closing the wound.

Statistical evaluation. To compare group mean body weights at the end of each of the dietary periods, $t$ tests were used.

Procedure. The rats were maintained on laboratory chow pellets (Tekland Mouse and Rat, $4.0 \%$ fat) ad lib prior to surgery. They were food and water deprived for $24 \mathrm{~h}$ before and after surgery. After surgery, they were first given the unadulterated highfat $(0.0 \%$ quinine) diet for 6 days to determine that all lesioned rats would display exaggerated body-weight gains. They then began maintenance on the quinine adulterated high-fat diets in order of increasing quinine concentration. The animals were maintained on each quinine diet until it appeared that both the VMH- and control-group body weights were relatively stable. Because consumption of adulterated diets is markedly influenced by body weight (Franklin \& Herberg, 1974; Maller, 1964), and because we anticipated that differences would exist in group body weights at the times the quinine content of the diet was increased, we did not measure food intake during this experiment.

Histology. Rats were anesthetized with chloroform and perfused intracardially with $.9 \%$ saline followed by $10 \%$ Formalin. Brains were stored in $10 \%$ Formalin until frozen sections were taken at 100 microns in the coronal plane described by König and Klippel (1963). Photographic enlargements of these unstained sections were used to assess the extent and location of tissue destruction.

\section{Results and Discussion}

Histological. The lesions generally destroyed much of the area bounded by the dorsomedial nuclei, the fornix, the anterior nuclei, and the premammillary nuclei. Damage to the premammillary nuclei was found in several animals, and these same animals showed sparing of the anteromost portions of the ventromedial nucleus (VMN). Damage normally ex- tended laterally to a point just ventral to or just past the plane of the fornix.

Behavioral. At the end of the $0.0 \%$ and $.4 \%$ periods, VMH rats weighed significantly more than control rats (ps $<.01$, Figure 1$)$. At the end of all other periods, there was no significant difference between the two groups. Figure 1 does not show the results obtained from the $4.0 \%$ period, since these results were confounded by the deaths of the two lightest animals in both the VMH and control groups. These four animals appeared to be eating little or none of the $4.0 \%$ quinine high-fat diet, and so their deaths are thought to be due to starvation rather than quinine toxicosis.

The failure to obtain significant mean differences during the $.6 \%$ and $.8 \%$ periods despite the large mean differences between the two groups was due to increasing variance among the VMH rats. One animal in that group, for example, continued to gain weight through the end of the $.8 \%$ period, while the others began losing weight, beginning with the switch from the $0.0 \%$ to the $.4 \%$ diet.

Despite increasing the concentration of quinine in the diet until deaths ensued in both control and lesioned groups, we were unable to drive the body weights of lesioned rats below those of controls. Adaptation to test solutions (Singh, 1974) or behavioral situations (King \& Gaston, 1973; Singh, 1973) before lesioning, however, can alter postsurgical behavior. In Experiment 1, the exposure to lower concentration quinine diets (e.g., .4\%, .6\%) that occurred after surgery might have served to adapt the animals to the quinine-adulterated diets, so mitigating effects that might otherwise have been seen at the higher concentrations. Experiment 2 was therefore conducted to test the effect of a high-concentration quinine diet on food intake and body weight without prior exposure to lower quinine concentration diets.

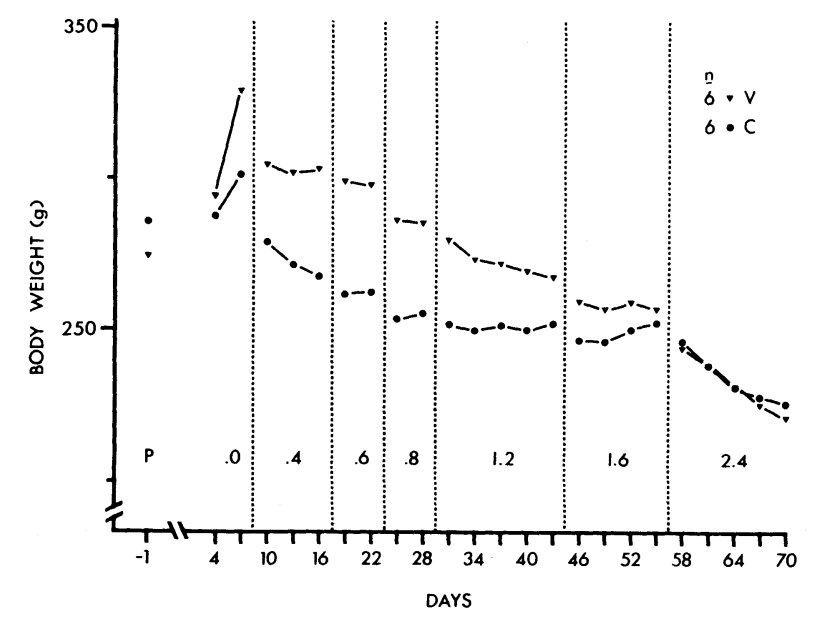

Figure 1. Group mean body weights. Abbreviations: $\mathrm{C}=$ control, $\mathbf{V}=\mathbf{V M H}$. 


\section{EXPERIMENT 2}

\section{Method}

Subjects. The subjects were 40 female Long-Evans hooded rats (Blue Spruce Farms, Inc.), weighing 160 to $218 \mathrm{~g}$ at the beginning of the experiment. Housing, surgical, and histological procedures were as in Experiment 1. Five rats died following surgery and one rat failed to become obese. The lesions in obese rats were generally as described in Experiment 1.

Diets. The unadulterated $(0.0 \%)$ and $1.6 \%$ quinine-adulterated high-fat diets from Experiment 1 were used and were presented as in Experiment 1. At the end of each 3-day period, body weight and 3-day cumulative food intake (spillage corrected) were measured. The $1.6 \%$ concentration was chosen because it was the concentration in Experiment 1 at which VMH and control groups maintained equivalent body weights with evidence of food consumption for all rats.

Statistical evaluation. To compare group mean body weights and food intakes, $t$ tests were used. The $t$ values obtained were compared with the conservative t' of Cochran-Cox (Kirk, 1968).

Procedure. All rats were given the unadulterated high-fat diet, beginning 7 days before surgery. The rats were food and water deprived for $24 \mathrm{~h}$ before and after surgery. After surgery, half of each surgical group continued maintenance on the unadulterated high-fat diet (CU, VU), while the other half of each surgical group began maintenance on the $1.6 \%$ quinine high-fat diet $(\mathrm{CQ}$, VQ). Beginning on Day 16, all rats were given the unadulterated high-fat diet to allow positive identification of obese rats in Group VQ.

\section{Results and Discussion}

During the course of access to the $1.6 \%$ quinine high-fat diet, VMH rats gradually lost more weight than control rats (Figure 2). On Day 16, VMH rats fed the quinine diet weighed significantly less than same-diet control rats (189.9 and $173.4 \mathrm{~g}$ for Groups $\mathrm{CQ}$ and $\mathrm{VQ}$, respectively; $\mathrm{t}=2.26, \mathrm{p}<.05$ ). Food intake data paralleled the body-weight data. Although both VMH and control rats initially consumed very little of the adulterated diet during the

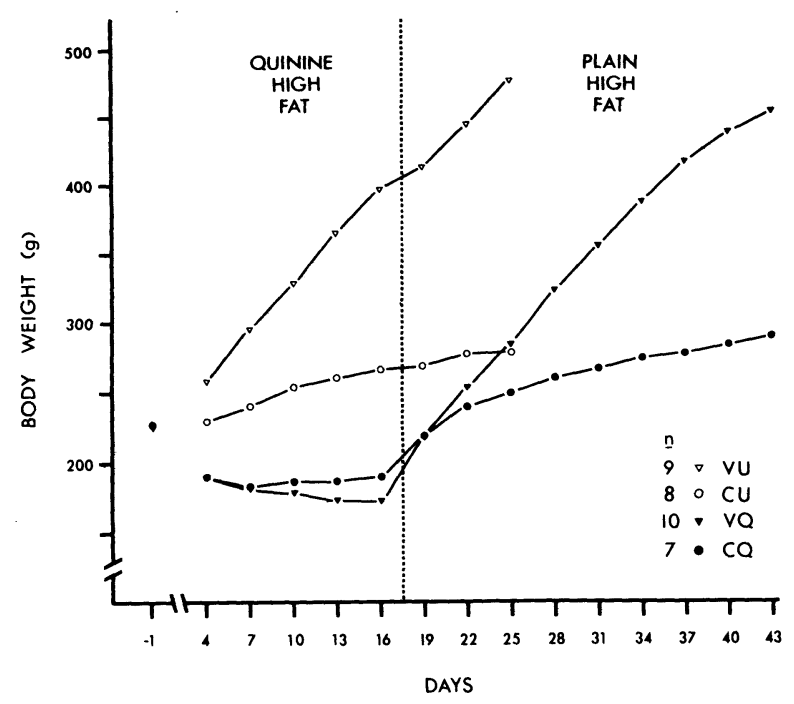

Figure 2. Group mean body weights. Abbreviations: $\mathbf{C}=$ control, $\mathbf{V}=\mathbf{V M H}, \mathbf{Q}=$ quinine adulterated diet, $\mathrm{U}=$ unadulterated diet. first two measurement periods (about 10-11 g means for both groups over 3-day periods compared to 40 and $80 \mathrm{~g}$ means for Groups CU and VU, respectively), the mean intake for control rats during Days 13-16 increased substantially to $23.3 \mathrm{~g}$, which was significantly $(t=2.12, p<.05)$ greater than the value for $\mathrm{VMH}$ rats $(15.4 \mathrm{~g})$.

When the groups were shifted to the unadulterated high-fat diet, VMH rats that previously had undereaten the $1.6 \%$ quinine diet and lost more weight than same-diet control rats displayed essentially the same rate of weight gain as VMH rats maintained on the unadulterated diet throughout. From Day 19, when Groups VQ and CQ had reached equal mean body weights, Group VQ gained an average of $9.9 \mathrm{~g}$ per day to attain a mean body weight of $455.7 \mathrm{~g}$ on Day 43. Corresponding values for Group CQ $(3.0$ and $291.1 \mathrm{~g})$ were significantly lower $(\mathrm{p}<.001)$.

The magnitude of the difference in mean body weight for Groups VQ and CQ on the last day of access to the $1.6 \%$ quinine high-fat diet was not large $(16.5 \mathrm{~g})$. Control rats fed this diet, however, weighed only $71 \%$ of the mean maintained by control rats fed the unadulterated diet, and the corresponding value for Group VQ was $65 \%$. Adulteration at concentrations much higher than $1.6 \%$ would probably decrease the mean difference in body weight between Groups VQ and CQ, because both groups would consume very little of the diet, that is, a floor effect. Adulteration at $1.6 \%$ was used for only 15 days in the present experiment because many VMH rats appeared severely debilitated.

\section{GENERAL DISCUSSION}

The general notion that obese rats with VMH lesions consume less food than control rats when diet palatability is substantially decreased is well documented (Powley, 1977). Ferguson and Keesey (1975) and Franklin and Herberg (1974), however, demonstrated that such rats undereat $.15 \%$ and $.2 \%$ quinine wet-mash diets only when their body weights exceed control levels. Undereating was attributed to obesity rather than VMH destruction.

The range of asymptotic body weights displayed by VMH rats after long-term access to diets that vary along a palatability dimension is much larger than the corresponding range for control rats. We (Peters et al., 1978) have argued, however, that the term "finickiness" should not be used to describe the feeding behavior of VMH rats simply because they display this expanded range. It is highly probable that many, if not all, experimental models of obesity display an expanded range of maintenance body weights across various palatability dimensions, because it is possible to adulterate any diet to make it basically inedible. The term "finickiness" would 
therefore apply to all such models and not be particularly meaningful for any.

In addition to an expanded range of body weight maintenance across a palatability dimension, both the data of Experiment 2 and our previous data (Peters et al., 1978) indicate that rats with VMH lesions eat and weigh less than same-diet control rats when fed certain adulterated diets. Similar data exist for rats with knife cuts between the medial and lateral hypothalamus (Gale \& Sclafani, 1977; Peters, Wellman, \& Gunion, 1979; Sclafani, Springer, \& Kluge, 1976). There are no such data, however, for other obesity syndromes, for example, ovariectomized rats (Gale \& Sclafani, 1977) or rats with dorsolateral tegmental lesions (Peters, Gunion, \& Wellman, 1979; Peters, Wellman, \& Gunion, 1979).

In summary, the present data provide a second demonstration that rats with VMH lesions eat and weigh less than same-diet control rats when fed a quinine-adulterated maintenance diet. Rats with VMH lesions both overeat and undereat diets that vary along a palatability dimension in a test situation in which undereating is not confounded with obesity. Within the stringent criteria we have used for the definition of finickiness, rats with VMH lesions can be described as finicky eaters. And, finally, we use the phrase descriptively, without any implication that finickiness causes the feeding disturbances of $\mathrm{VMH}$ rats. Overeating and undereating may be dissociable phenomena (Graff \& Stellar, 1962).

\section{REFERENCES}

Ferguson, N. B. L., \& Keesey, R. E. Effect of a quinineadulterated diet upon body weight maintenance in male rats with ventromedial hypothalamic lesions. Journal of Comparative and Physiological Psychology, 1975, 89, 478-488.

Franklin, K. B. J., \& Herberg, L. J. Ventromedial syndrome: The rat's "finickiness" results from the obesity, not from the lesions. Journal of Comparative and Physiological Psychology, 1974, 87, 410-414.

Gale, S. K., \& Sclafani, A. Comparisons of ovarian and hypothalamic obesity syndromes in the female rat: Effects of diet palatability on food intake and body weight. Journal of Comparative and Physiological Psychology, 1977, 91, 381-392.

GrafF, H., \& Stellar, E. Hyperphagia, obesity and finickiness. Journal of Comparative and Physiological Psychology, 1962, 55, 418-424.

King, B. M., \& Gaston, M. G. The effects of pretraining on the bar-pressing performance of VMH-lesioned rats. Physiology \& Behavior, 1973, 11, 161-166.

KIRK, R. E. Experimental design: Procedures for the behavioral sciences. Belmont, Calif: Brooks/Cole, 1968.

König, J. F. R., \& KLIPPEL, R. A. The rat brain. Baltimore: Williams and Wilkins, 1963.

Malle R, O. The effect of hypothalamic and dietary obesity on taste preference in rats. Life Sciences, 1964, 3, 1281-1291.

Miller, N. E., Bailey, C. J., \& Stevenson, J. A. F. Decreased "hunger" but increased food intake resulting from hypothalamic lesions. Science, 1950, 112, 256-259.

Peters, R. H., Gunion, M. W., \& Wellman, P. J. Influence of diet palatability on maintenance feeding behavior in rats with dorsolateral tegmental damage. Physiology \& Behavior, 1979, 23, 685-692.

Peters, R. H., Luttmers, L. L., Gunion, M. W., \& Wellanan, P. J. Ventromedial hypothalamic syndrome: Finickiness? Physiology \& Behavior, 1978, 20, 279-285.

Peters, R. H., Wellman, P. J., \& Gunion, M. W. Experimental obesity syndromes in rats: Influence of diet palatability on maintenance body weights. Physiology \& Behavior, 1979, 23, 693-699.

Powley, T. L. The ventromedial hypothalamic syndrome, satiety, and a cephalic phase hypothesis. Psychological Review, 1977, 84, 89-126.

Sclafani, A., Springer, D., \& Kluge, L. Effects of quinine adulterated diets on the food intake and body weight of obese and non-obese hypothalamic hyperphagic rats. Physiology \& Behavior, 1976, 16, 631-640.

SingH, D. Effects of preoperative training on food-motivated behavior of hypothalamic hyperphagic rats. Journal of Comparative and Physiological Psychology, 1973, 84, 45-52.

SingH, D. Role of preoperative experience on reaction to quinine taste in hypothalamic hyperphagic rats. Journal of Comparative and Physiological Psychology, 1974, 86, 674-678.

Teitelbaum, P. Sensory control of hypothalamic hyperphagia. Journal of Comparative and Physiological Psychology, 1955, 48, 156-163.

Wellman, P. J., \& Peters, R. H. Effects of cellulose adulteration on maintenance-feeding behavior in rats with VMH lesions. Physiological Psychology, 1978, 6, 493-496.

(Received for publication November 20, 1979; accepted January 30, 1980.) 